\title{
Gleaning Secrets from the Transverse Profiles of AGN jets
}

\author{
Eoin Murphy* \\ University College Cork, Ireland \\ E-mail: eoin.g.murphy@gmail.com
}

\section{Denise Gabuzda}

University College Cork, Ireland

E-mail: d.gabuzda@ucc.ie

Tim Cawthorne

University of Central Lancashire, England

E-mail: tvcawthorne@uclan.ac.uk

\begin{abstract}
Both the emission properties and evolution of Active Galactic Nuclei (AGN) radio jets are dependent on the magnetic fields that thread them. A better understanding of these magnetic fields is therefore important in helping our understanding of jets in AGN. Several observations of jets have suggested that, on parsec scales, the jet magnetic field may have a significant helical component. Using a model first proposed by Laing and developed by Papageorgiou and Cawthorne, all of the above observations can be reproduced by varying only the helical pitch angle of the magnetic field and the line of sight angle. In order to reduce the total polarization to agree with observed values, a tangled magnetic field component is also introduced to the model, via another parameter, the degree of entanglement. We are in the process of comparing data from observations of several AGN with this model, making it possible to derive estimates of the helical pitch angle, the viewing angle and the degree of entanglement for these jets in the framework of this model. The observed profiles are compared with a set of theoretical profiles. This proves to be an objective approach to profile fitting that should enable analysis of a large number of AGN jets, making it possible to look for trends. Results for Mrk501 are presented
\end{abstract}

10th European VLBI Network Symposium and EVN Users Meeting: VLBI and the new generation of radio arrays

September 20-24, 2010

Manchester, $U K$

\footnotetext{
* Speaker.
} 


\section{Introduction}

At radio wavelengths the jets of active galaxies emit synchrotron radiation, which occurs when charged particles move relativistically through magnetic fields. This is characterised by appreciable linear polarization with the plane of the polarization perpendicular to the plane of the jet magnetic field in the optically thin region. The polarization structure of the jets provides information about the structure of the magnetic fields threading these jets. In order to understand both the evolution and emission properties of AGN an understanding of the magnetic fields that thread them is important. Yet despite much observational effort the nature of the magnetic field structures of AGN are not well understood. Several observational results for jets have suggested that the magnetic field threading the jet may have a significant helical component on parsec scales. $[3,4,5]$

There are primarily 3 different types of observational results that support the theory that the jets of AGN are threading by helical magnetic fields, all of which have been observed in the jets of a number of AGN.

- Asymmetric profiles of total intensity and polarization across jets.

- Magnetic field orientation changing from longitudinal to transverse within a given jet profile.

- Systematic Faraday Rotation Measure gradients across jets.

These properties can also be attributed to physical asymmetries, such as differing pressures and surrounding jet environments on either side of the jet [1]. Examples include edge brightening due to compression on one side of the jet and a change of the magnetic field orientation from transverse to longitudinal due to sheering between the jet and external medium. However, a helical magnetic field threading the jet of an AGN can elegantly describe all these observations while requiring no further phenomena to be occurring in the jet environment.

\section{Helical Magnetic Field Model}

The model we have used consists of a helical magnetic field of constant pitch angle and uniform flux density threading a cylindrical jet. This model geometry was first proposed by Laing [2] and further investigated by Papageorgiou [3]. It makes predictions of both the total intensity and polarized intensity distributions across a jet using only 2 parameters, the helical pitch angle $\gamma$ and the viewing angle $\delta$.

This model produces 4 different magnetic field configurations, as is shown in Figure 1 (from top to bottom).

1. Longitudinal all across the jet

2. Longitudinal on one side and transverse on the other

3. Longitudinal at the edges and transverse at the centre

4. Transverse all across 
Configuration 2 only occurs when $\gamma=\delta$ and configuration 4 only occurs when $\gamma=\delta=90$ degrees. These special cases would not be expected to be observed often in nature. However, these theoretical profiles assume infinite resolution. Mimicing the effects of finite resolution by convolving the profiles with a Gaussian beam of a suitable full width at half maximum (FWHM) increases the range of parameter values for which the different polarization configurations are observed.

Observations analysed by Papageorgiou [3] showed that this model produced polarized intensity distributions which were much greater than observed. The model was accordingly modified by introducing a disordered, tangled magnetic field component. As result the magnetic field geometry is described as the sum of two components; one corresponding to a helical field, as described by Laing [2], and a second, corresponding to a field that is tangled on angular scales much smaller than the width of the jet. Introducing this tangled magnetic field introduces a third parameter to the model, the degree of disorder, $f$. This parameter is defined as the fraction of the magnetic field energy density in tangled form.

$$
\frac{\left\langle B_{T}^{2}\right\rangle}{\left\langle B_{H}^{2}\right\rangle}=\frac{f}{1-f}
$$

where $\left\langle B_{T}^{2}\right\rangle$ and $\left\langle B_{H}^{2}\right\rangle$ are the magnetic field energy densities of the tangled field and the helical field respectively. Increasing the degree of disorder, $\mathrm{f}$, decreases asymmetries in the total intensity profiles generated by the model in addition to decreasing the degree of polarization.

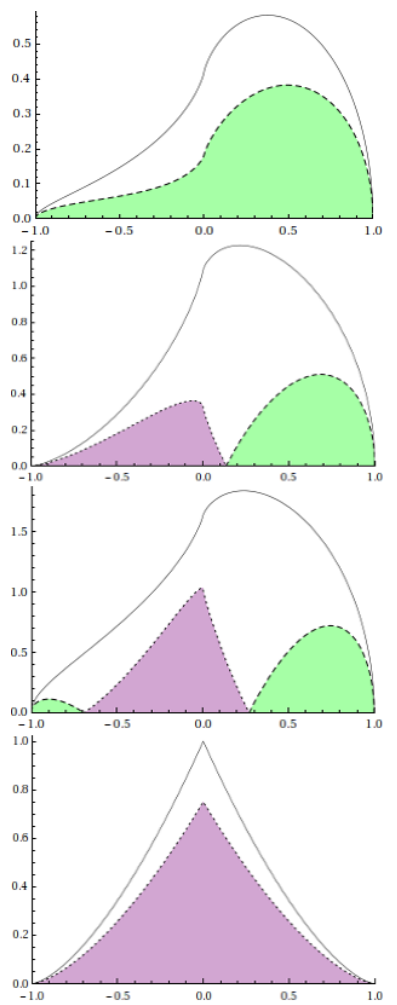

Figure 1: Theoretical profiles for every magnetic field distribution. Solid line corresponds to total intensity, purple region to expected transverse polarization and green region to expected longitudinal polarization.

\section{Method of Comparison}

For comparison with the observed profiles a database of theoretical profiles was generated. The database profiles were convolved with a Gaussian of FWHM equal to that of the beam used in the observations. The best fit profile is determined via a $\chi^{2}$ minimisation. The database profiles are scaled so that the maximum total intensity of the theoretical profile is equal to the maximum total intensity of the observed profile. As the range of parameters that can reproduce a total polarization distribution is much smaller than the range of values that can reproduce a total intensity distribution the total polarization profile were given more weight than the total intensity profile in the comparison algorithm. Errors are calculated by changing one of the parameters in steps from it's best fit value and allowing the other 2 parameters to vary, until the $\chi^{2}$ value increases by unity compared to the best fit $\chi^{2}$. This is continued until such a fit cannot be found. Repeating this procedure gives $1 \sigma$ errors for all 3 parameters. 
The total time taken for this method to find the best fit values of $\gamma, \delta$ and $f$ and their corresponding errors is approximately 20 minutes per profile (for a $3 \mathrm{GHz}$ Dual Core processor and $3.2 \mathrm{~GB}$ of RAM) . The time required for the algorithm is due to the fact that it carries out an exhaustive search for the $\chi^{2}$ minimum. However, it's advantages are that it provides an objective way to fit multiple profiles for a single source or profiles for multiple sources, and also provides information about secondary local minima.

\section{Markarian 501}

VLBI images of Markarian 501 (also known as DA426 or 1652+398) often show excellent examples of sudden magnetic field orientation changes across the jet [4]. Figure 2 shows the $6 \mathrm{~cm}$ polarization map of this source for the data of [4]. Three slices were taken across this map using the SLICE task in the NRAO AIPS package. Both Slice 1 (Orange) and Slice 3 (Green) were taken in regions of the jet without any unusual polarization structure. Slice 2 (Yellow) was taken in the region of sudden magnetic field orientation changes. These slices were compared to a database of theoretical slices and the values of $\gamma, \delta$ and $\mathrm{f}$ which resulted in the best fit (minimum $\chi^{2}$ ) were found, presented in Table 1. The observed profile and the model's best fit profile for Slice 2 can be seen in Figure 3. The overall consistency of the fitted $\gamma, \delta$ and for the three slices is strik-

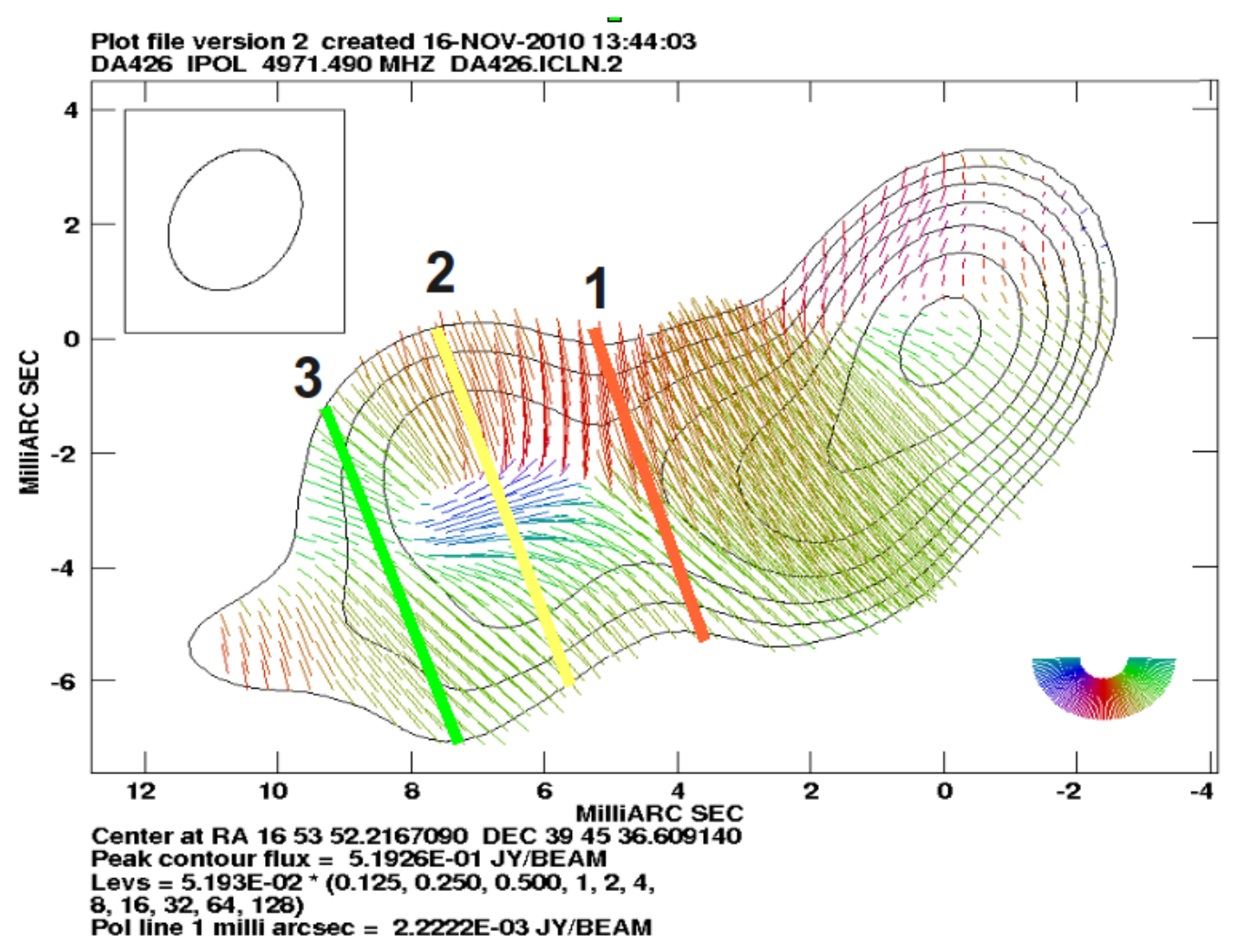

Figure 2: $6 \mathrm{~cm}$ Polarization Map of Mrk501 [4]. Magnetic field orientation is perpendicular to polarization lines. The 3 lines across the jet show the 3 transverse slices that were analysed. 
Table 1: Best fit Parameters for $6 \mathrm{~cm}$ Mrk501 Slices.

\begin{tabular}{|c|c|c|c|}
\hline Slice & $\gamma$ & $\delta$ & f \\
\hline Slice 1 & $49_{-4}^{+11}$ Degrees & $78_{-1}^{+2}$ Degrees & $0.40_{-0.13}^{+0.10}$ \\
\hline Slice 2 & $53_{-3}^{+7}$ Degrees & $80_{-4}^{+1}$ Degrees & $0.40_{-0.09}^{+0.31}$ \\
\hline Slice 3 & $58_{-5}^{+11}$ Degrees & $78_{-1}^{+3}$ Degrees & $0.80_{-0.33}^{+0.12}$ \\
\hline
\end{tabular}

ing. These results show tentative evidence that the pitch angle of the helical field threading this jet increases with distance from the core, consistent with the expectation that the toroidal field component should become more dominant with distance.

Table 2 shows our best fit parameters for slices taken at four different wavelengths: the $4 \mathrm{~cm}$ and $6 \mathrm{~cm}$ maps of Pushkarev et al [4] for February 2007, and the $13 \mathrm{~cm}$ and $18 \mathrm{~cm}$ maps of Croke at al [5] for May 1998. These 4 slices were all taken in the same region of the jet, specifically the region of Slice 2 in Figure 2 The derived parameters are consistent for the slices in the same region of the jet observed at $4 \mathrm{~cm}, 6 \mathrm{~cm}, 13 \mathrm{~cm}$ and $18 \mathrm{~cm}$ (at two different epochs).

It is important to note that the viewing angle of the jet determined by this method is the viewing angle in the rest frame of the jet. The viewing angle that we observe $\delta^{\prime}$ is given by:

$$
\delta^{\prime}=\frac{\delta}{\Gamma(1-\beta \cos \delta)}
$$

where $\Gamma$ is the Lorentz factor of the jet and $\beta=\frac{v}{c}$. For $\delta$ close to 90 degrees this simplifies to $\delta^{\prime} \approx \frac{\delta}{\Gamma}$.

\section{Conclusion}

Using a relatively simple model for a helical magnetic field threading an AGN jet it is possible to derive estimates of the helical pitch angle, the viewing angle and the degree of entanglement for these jets (in the framework of this model). Preliminary results for Mrk501 demonstrate a consistency in these estimates for both slices across different regions at one frequency and slices across the same region at different frequencies. These results suggest that this jet carries a helical B field with pitch angle $\gamma \simeq 55$ degrees viewed at an angle of $\delta \simeq 85$ in the jet rest frame, and provide tentative evidence that the pitch angle of the helical field threading this jet increases with distance

Table 2: Best fit Parameters for Mrk501 Slices at 4 different frequencies.

\begin{tabular}{|c|c|c|c|c|}
\hline Slice & $\gamma$ & $\delta$ & f & Epoch \\
\hline $4 \mathrm{~cm}$ & $53_{-1}^{+1}$ Degrees & $83_{-3}^{+1}$ Degrees & $0.40_{-0.04}^{+0.05}$ & February 1997 \\
\hline $6 \mathrm{~cm}$ & $53_{-3}^{+7}$ Degrees & $80_{-4}^{+1}$ Degrees & $0.40_{-0.09}^{+0.31}$ & February 1997 \\
\hline $13 \mathrm{~cm}$ & $54_{-2}^{+1}$ Degrees & $85_{-5}^{+1}$ Degrees & $0.45_{-0.05}^{+0.02}$ & May 1998 \\
\hline $18 \mathrm{~cm}$ & $53_{-1}^{+2}$ Degrees & $86_{-4}^{+2}$ Degrees & $0.45_{-0.26}^{+0.02}$ & May 1988 \\
\hline
\end{tabular}




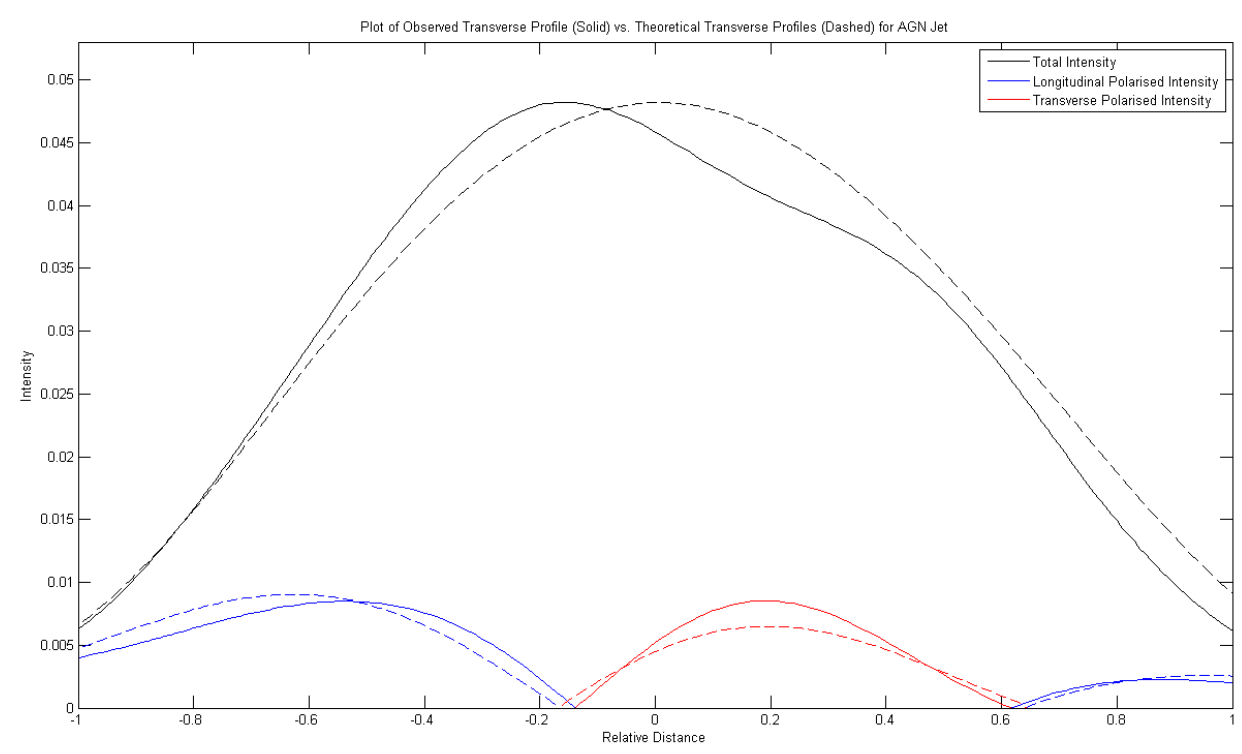

Figure 3: Plot of model fit for Slice 2 of $6 \mathrm{~cm}$ MK501 map for $\gamma=53$ degrees, $\delta=80$ degrees and $\mathrm{f}=$ 0.4. Solid line corresponds to observed profile while dashed line corresponds to model profile. Blue lines correspond to longitudinal magnetic field orientation while red lines correspond to transverse magnetic field orientation. The polarization has been scaled by a factor of 3 .

from the core.

Future work involves applying this method to different sources and devising a way to ensure the observed profiles are taken perpendicular to the jet direction. We also hope to compare observed Faraday rotation measure gradients to gradients generated by this model.

\section{Acknowledgements}

Funding for this research was provided by the Irish Research Council for Science Engineering and Technology (IRCSET).

\section{References}

[1] Attridge J. M., Roberts D. H., Wardle J. F. C., 1999, ApJ, 518, 87

[2] Laing R. A., 1981, ApJ, 248, 87

[3] Papageorgiou A, 2005, PhD. Thesis

[4] Pushkarev A. B., Gabuzda D. C., Vetukhnovskaya Yu. N.,Yakimov V. E. 2005, MNRAS, 356, 859

[5] Croke S.M., O'Sullivan S.P., Gabuzda D.C., 2010, MNRAS, 402,259 